\title{
Letter
}

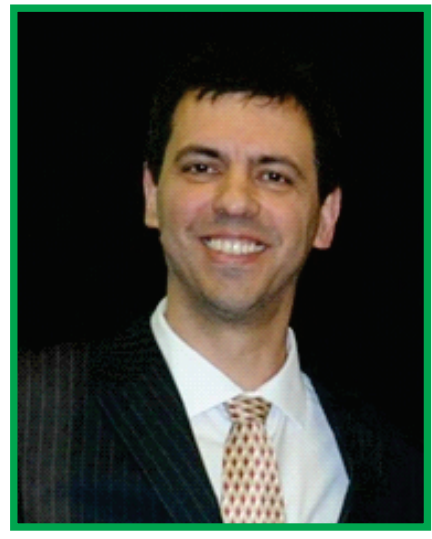

\section{Nanoscience and Nanotechnology - A True Revolution in the Way of Thinking Nature}

\author{
Aldo José Gorgatti Zarbin \\ Full Professor - Materials Chemistry Group (GQM) \\ Department of Chemistry - Federal University of Paraná (UFPR) \\ Centro Politécnico, Curitiba, PR, Brazil \\ aldozarbin@ufpr.br
}

The scientific knowledge produced in the last decades of the $20^{\text {th }}$ century were strongly characterised by coming and the consolidation of a novel way to treat the matter, reaching for an understanding of the innovative phenomena and properties arising from the bottom. Nanoscience and Nanotechnology (N\&N) represented a true revolution in the way of thinking nature, and brought us a myriad of possibilities for the application of so-called nanomaterials in novel technological devices and systems.

Nanoscience can be understood as the science dealing with the phenomenon whereby the properties of materials are strongly dependent on their size. Materials with particles below a critical size - in the range of nanometres - present properties that are different from the same materials as a bulk, which means that unique materials (and new properties) can be designed simply by selecting different particle sizes. Notwithstanding the paper published by Michael Faraday in 1857, in which the different colours of colloidal gold (blue, purple, green) were associated with the different sizes of gold particles (which in the personal opinion of the signatory of this letter represents the outset of the $N \& N$ ), or the lecture given by Richard Feynman during the $29^{\text {th }}$ Meeting of the American Physical Society in 1959 calling on scientists to dedicate more efforts to the understanding of matter at very small sizes, the relationship between size and properties started to become important only at the end of the 1980s, reaching a boom in the last decade of the twentieth century that remains to date. Nanoscience brought with it nanotechnology, in which products, systems and devices produced by it represent the fastest growing market in the world nowadays. The global nanotechnology market was approximately US\$ 39.2 million in 2016 and is expected to reach 90.5 billion by 2021 and exceed 125 billion by 2024, according the Research and Markets company report released in 2018.

Nanoscience and nanotechnology represent a perfect example of inter- and trans-discipline fields. It is not excessive to say that N\&N passes through all of the knowledge areas and directly impacts almost all fields: energy, medicine, agriculture, environment, goods and services, manufacturing, personal care, health, electronics, textiles, chemicals, computers, materials, communication, transportation, defence, entertainment... and so on. As expected, chemistry has played a central role in all of the steps of N\&N development and consolidation. First, by solving the core problem related to the synthesis of stable nanomaterials. Chemists have been developing several creative strategies to work around the intrinsic thermodynamic instability of nanomaterials, and have making the preparation of different materials in a nanometric scale possible, with rigorous control of the size dispersion and stability. Second, chemists have been working together with physicists, engineers, biologists, material scientists, and mathematicians, among others, to help to understand the physical phenomena behind the experimental data, as well as the development of novel and sophisticated characterisation techniques.

Nanochemistry belongs to all the traditional subfields of chemistry, with analytical chemistry being one of the most participative. The global community of analytical chemists quickly understood the importance 
and infinite number of possibilities of that emerging knowledge and incorporated it to their day-to-day work. Novel sensors showing unbelievable detection limits of single molecules have been produced; classical problems have been solved, multi-detection techniques have been developed, and a new challenge has been proposed: the detection and understanding of the environmental and health issues directly associated with these novel classes of nanomaterials.

Following the global tendency, Brazilian analytical chemists have also been presenting important contributions in the N\&N, and this special edition of the Brazilian Journal of Analytical Chemistry is a clear demonstration of that. As a materials chemist who has dedicated his entire scientific work to different aspects of the synthesis, characterisation and application of nanomaterials (including several directly related to Analytical Chemistry), I am very proud to recognise the strength of this community, as well as the beauty, originality and relevance of nanoscience and nanotechnology associated with analytical chemistry developed in Brazil. Congratulations to the editors for the initiative. 